\title{
Spectral change detection for deep-hole drilling
}

\author{
Anita S. Busch and Ursula Gather
}

A control chart for detecting changes in the spectrum of a time series is developed. It is based on a spectral representation using the SLEX function. The control chart compares spectral characteristics from recent to past time windows with spectral divergence measures. As an application, the structure-borne sound measured at the single-lip deep-hole boring machine is put under statistical investigation in order to detect signs of chatter vibration in the process. Different spectral divergence measures are empirically compared with respect to their ability to separate non-chattering from chattering process parts.

Keywords AND PHRASEs: Chatter detection, Control chart, Exponential weighting, Smooth localized exponential, Spectral divergence measures.

\section{INTRODUCTION}

We consider the single-lip deep-hole drilling, a method yielding deep holes with small diameter with high surface quality and little straightness deviation, e.g. for valve guides (Weinert, Peters, and Mehnen 2002). However, because of the large length-to-diameter ratio of the tool and its asymmetric appearance with only one cutting edge the process is extremely unstable and susceptible to dynamic disturbances, such as tool chatter, which can result in poor drillhole quality. This also affects tool and machine life. It is our aim to reliably detect structural changes in the sensor signal by means of appropriately designed statistical change detection schemes. The signal of the structure-borne sound of the machine measured with $20000 \mathrm{~Hz}$ carries the sufficient information for detecting chatter vibration in the process especially when looking at its spectrum (Weinert et al. 2002).

Here, SLEX periodograms (cf. Section 2) are used which can give a time and frequency localized representation of all relevant patterns without redundancies as suggested by Ombao, Raz, von Sachs, and Malow (2001). They have derived a data adaptive time varying spectral density estimator based on the SLEX function. For an online analysis we are faced with the difficulty that most methods for detecting changes mentioned in the literature assume abrupt changes. Here however, a lot of different types of changes - mostly of gradual type - have to be detected. Moreover, changes due to chattering have to be distinguished from small changes in states of steady boring.

There exist different approaches mainly working in the frequency domain of a time series. Parametric approaches to an online analysis are described in Basseville and Nikiforov (1993) and Ombao, Heo, and Stoffer (2004). In our application however, weaker model assumptions are preferable. The offline method of Picard (1985) uses the Kolmogorov metric as spectral distance measure and is explicitly constructed for abrupt changes. This idea is also used in Ligges, Weihs, and Hasse-Becker (2002) for an online procedure based on comparisons of different small disjoint time windows. The Kolmogorov metric, however, works particularly well if power changes in one frequency only. Another interesting control chart method using the evolutionary spectrum of Priestley (1988) is described in Subba Rao (1981). There, points in time and frequency are chosen as far apart that the corresponding estimates of the spectrum are approximately uncorrelated. For each time point, the sum of the logarithm of these values provide the basis for the construction of a univariate CUSUM control chart (Page, 1961). Thus, the resulting metric for comparing time windows reduces to an absolute difference of measures of dispersion.

The above mentioned methods are useful in many applications, but they are not suitable for the kind of data from single-lip deep-hole drilling. Either they do not work online, or they only detect abrupt changes. If gradual changes can be detected at all, small irrelevant but steady changes are ignored. A further problem is that most of the spectral divergence measures used in the procedures are not suitable or sensitive enough to separate sequences in the singlelip drilling process with chatter from those without. Hence, naïve control charts are not successfully applicable here. In fact, an entirely new strategy has to be developed to achieve the specific aim of finding gradual changes in the spectrum of a non-stationary time series.

We will discuss which spectral distortion measures can be used alternatively to compare the weighted mean of the spectral density estimates with a reference distribution. For this purpose, an extensive empirical comparison of different divergence measures is carried out in Section 3. We present a nonparametric method for the detection of spectral changes in the structure-borne sound signal in Section 4. This control chart is a modified version of the multivariate EWMA chart as described in Lowry, Woodall, Champ, and Rigdon (1992). It is based on the values of the SLEX periodograms calculated in overlapping windows over time and uses a more suitable divergence measure than the Mahalanobis distance. A further modification step is necessary to ensure that small 
gradual changes in the spectrum of signals from processes without chatter vibration do not cause false alarms. An application of the chart is shown in Section 5 and a summary of the results is given in Section 6 .

\section{THE SLEX PERIODOGRAM}

Consider a time series $\left\{X_{t}\right\}_{t \in \mathbb{Z}}$ with $\mathrm{E}\left(X_{t}\right)=0$ for all $t \in \mathbb{Z}$. Ombao et al. (2001) introduce the smooth localized exponential (SLEX) transform as a special case of the smooth localized orthogonal basis functions of Wickerhauser (1993). The SLEX basis functions

$$
\varphi_{\omega}(t)=\vartheta^{+}(t) e^{i t \omega}+\vartheta^{-}(t) e^{-i t \omega}, \quad t \in \mathbb{Z}, \omega \in(-\pi, \pi]
$$

are defined such that they are simultaneously orthogonal and localized not only in frequency but also in time. Localization in time and frequency is thoroughly described in Carmona, Hwang, and Torrésani (1998) and is an important property for the spectrum to be interpretable.

We assume when working with discrete observed time series, that the two real window functions $\vartheta^{+}$and $\vartheta^{-}$have support $\{-m+1, \ldots, m\}$ with $m \in \mathbb{N}$, where $\vartheta^{+}$looks like a usual taper function, whereas the window function $\vartheta^{-}$is effective (nonzero) at the time points where $\vartheta^{+}$is weighted down.

With an arbitrary starting point followed by $\varepsilon \in \mathbb{N}$ time points, we carry out a segmentation of the rest of the time series into segments of length $N, N$ even, which we denote by an index $k \in \mathbb{N}$. Then the windows $\vartheta^{+}$and $\vartheta^{-}$of window width $2 m$ with $m=N / 2+\varepsilon$ and centers equal to the centers of the segments are considered. The SLEX periodogram corresponding to the time series segment with index $k$ can be defined as

$$
I_{k}\left(\omega_{j}\right)=\frac{1}{N}\left|\sum_{t=-m+1}^{m} \varphi_{\omega_{j}}^{*}(t) X_{t+m+(k-1) N}\right|^{2},
$$

with $\omega_{j}=\frac{2 \pi j}{N}, j \in\{-N / 2+1, \ldots, N / 2\}$. Thus, the time series segments for $I_{k}\left(\omega_{j}\right)$ and $I_{k+1}\left(\omega_{j}\right)$ overlap by $2 \varepsilon$ time points. The region where the windows overlap is the part which is downweighted in $\vartheta^{+}$. Nevertheless, $I_{k}\left(\omega_{j}\right)$ and $I_{k+1}\left(\omega_{j}\right)$ are asymptotically uncorrelated if $\left\{X_{t}\right\}_{t}$ is a stationary process.

Theorem 1. Let $\left\{X_{t}\right\}_{t \in \mathbb{Z}}$ be a stationary Gaussian process with $\mathrm{E}\left(X_{t}\right)=0$ for all $t \in \mathbb{Z}$. Let the spectral density of the process be $f(\omega)=f(-\omega), \omega \in(-\pi, \pi]$, and let the covariance structure of the process satisfy the usual convergence assumptions (Brillinger 1981, p. 92). Furthermore, let $I_{k}\left(\omega_{j}\right)$ be the SLEX periodogram of the $k$-th segment of a partition of an arbitrary part of the series with segments of length $N$ and let $\omega_{j}$ be the $j$-th Fourier frequency with respect to $N$. Then

$$
\operatorname{Cov}\left(I_{k}\left(\omega_{j}\right), I_{h}\left(\omega_{j}\right)\right)=\mathrm{O}\left(1 / N^{2}\right)
$$

for all $k \neq h, k, h \in \mathbb{N}, j \in\{-N / 2+1, \ldots, N / 2\}$, and

$$
\operatorname{Cov}\left(I_{k}\left(\omega_{j}\right), I_{k}\left(\omega_{i}\right)\right)=\mathrm{O}(1 / N)
$$

for all $i \neq j$ with $i, j \in\{-N / 2+1, \ldots, N / 2\}, k \in \mathbb{N}$.

The proof is given in Appendix A.

By plotting the SLEX spectrogram we now investigate a time series of the structure-borne sound from a process where a drilling expert noticed a slight chatter vibration in the second half of the process. Therefore, we apply a SLEX transform and construct SLEX periodograms using a partition with segments of length $N=1024$. We get Figure 1 as a representation of the time dependent spectrum of this time series. It is quite similar to an ordinary spectrogram but has the advantage that because of the construction with basis functions no information is lost nor are there any redundancies. The area in which a slight chatter is recognized is located between 30 and $50 \mathrm{~mm}$ boring depth and in some frequency bands the power increases gradually. Here and in other processes we notice that in calm sequences changes in the spectrum are present. That means chattering is interpreted as a too big change compared to the current state of the spectral characteristics of the process. Strong chatter mostly occurs abruptly along with a strong rise of the amplitude of the structure-borne sound. This type of chatter can be provoked by high cutting and feeding speeds and are easily detected by simple methods. The challenge is here to detect slight chatter coming along with a gradual or jiggling rise of the power in some frequencies of the spectrum. In Section 4 we use the SLEX periodogram to construct a control chart explicitly constructed for this purpose.

\section{SPECTRAL DIVERGENCE MEASURES}

In this section some spectral distances and divergences based on spectral density estimates are compared. Let $\hat{f}$ and $\hat{g}$ be estimators of spectral densities $f$ and $g$ belonging to two different stationary processes based on observations with the same length $n, n$ even for convenience. We define the following measures as the divergences between the estimates at the Fourier frequencies $\omega_{j}=2 \pi j / n, j \in\{-n / 2+1, \ldots, n / 2\}$. We may concentrate on $j \in\{1,2, \ldots, n / 2\}$ because if $X_{t}$ is real for all $t$ periodograms and other spectral density estimates are symmetric in general and if $\mathrm{E}\left(X_{t}\right)=0$ for all $t$ we get $f(0)=0$. Corresponding measures for the theoretical spectral densities can be found e.g. in Basseville (1989), and Gray and Markel (1976). A new class of divergence measures, however with special emphasis on testing partial coherences, is discussed in Eichler (2007).

The Itakura-Saïto divergence, related to the KullbackLeibler information criterion (see Busch, 2006),

$$
d_{\mathrm{IS}}(\hat{f}, \hat{g})=\frac{2 \pi}{n} \sum_{j=1}^{n / 2}\left(\frac{\hat{f}\left(\omega_{j}\right)}{\hat{g}\left(\omega_{j}\right)}-\ln \frac{\hat{f}\left(\omega_{j}\right)}{\hat{g}\left(\omega_{j}\right)}-1\right),
$$




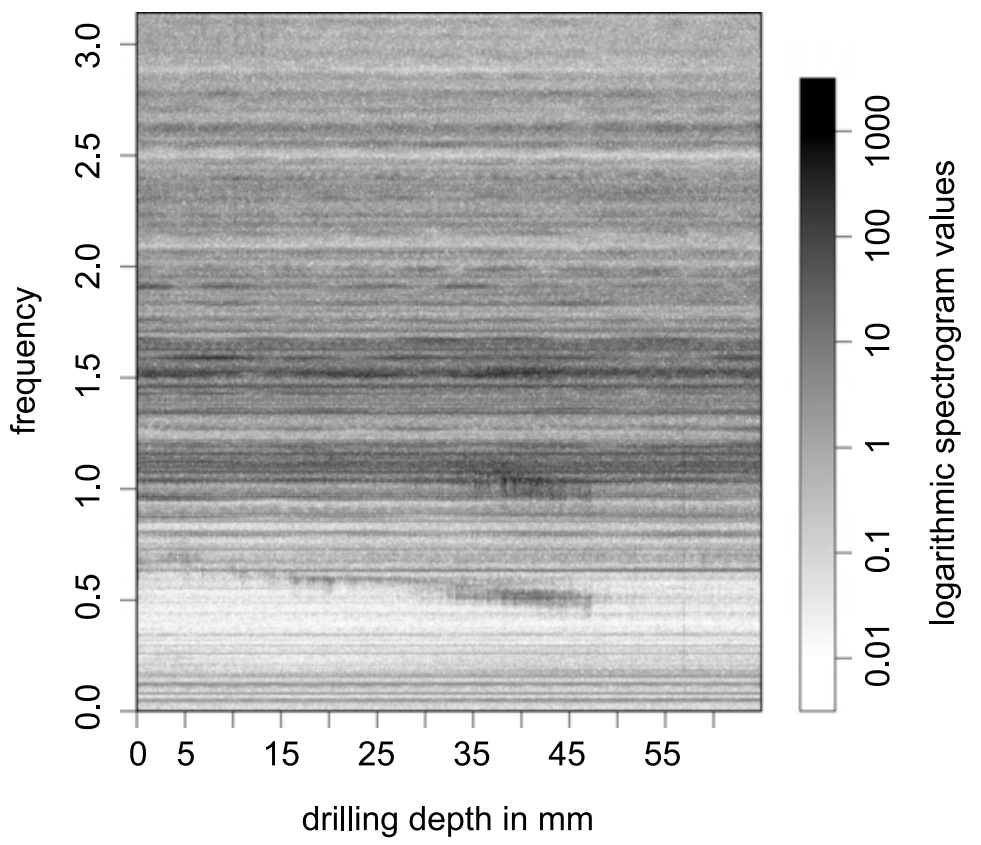

Figure 1. SLEX Spectrogram for the Time Series of Structure Borne Sound from a Drilling Process with Slight Chatter Vibrations: $n \approx 600000,64+1024+64$ Data Points per Window, 512 Frequencies.

its symmetric version

$$
d_{\mathrm{ISs}}(\hat{f}, \hat{g})=\frac{\pi}{n} \sum_{j=1}^{n / 2}\left(\frac{\hat{f}\left(\omega_{j}\right)}{\hat{g}\left(\omega_{j}\right)}+\frac{\hat{g}\left(\omega_{j}\right)}{\hat{f}\left(\omega_{j}\right)}-2\right),
$$

and the $L_{p}$-norm applied to the difference of the logarithmic estimated spectral density

$$
d_{\mathrm{lLp}}(\hat{f}, \hat{g})=\frac{2 \pi}{n} \sqrt[p]{\sum_{j=1}^{n / 2}\left|\ln \hat{f}\left(\omega_{j}\right)-\ln \hat{g}\left(\omega_{j}\right)\right|^{p}}
$$

are all spectral divergence measures. The Kolmogorov metric for differences of the empirical spectral distribution functions

$$
d_{\mathrm{KA}}(\hat{f}, \hat{g})=\frac{4 \pi}{n} \max _{j}\left|\sum_{i=-n / 2+1}^{j} \hat{f}\left(\omega_{i}\right)-\sum_{i=-n / 2+1}^{j} \hat{g}\left(\omega_{i}\right)\right|,
$$

is used in the offline test of Picard (1985), and in the online change detection scheme of Ligges et al. (2002). The absolute difference of the sum of logarithmic spectral estimates

$$
d_{\mathrm{DIL}}(\hat{f}, \hat{g})=\frac{2 \pi}{n}\left|\sum_{j=1}^{n / 2} \ln \hat{f}\left(\omega_{j}\right)-\sum_{j=1}^{n / 2} \ln \hat{g}\left(\omega_{j}\right)\right|
$$

is a similar measure as the one used in the control chart by Subba Rao (1981). The difference of the sums of all spectral density estimate values

$$
d_{\operatorname{DeV}}(\hat{f}, \hat{g})=\frac{2 \pi}{n}\left|\sum_{j=1}^{n / 2} \hat{f}\left(\omega_{j}\right)-\sum_{j=1}^{n / 2} \hat{g}\left(\omega_{j}\right)\right|
$$

corresponds to the difference of the empirical variances when $\hat{f}$ and $\hat{g}$ are periodograms.

In an empirical study we compare the different spectral divergence measures in order to investigate their ability to distinguish between the relations among two chatter-free and among one chatter-free and one chattering process sequence. For the estimation of the spectral measures used in the discrepancies different strategies are used and compared:

- As spectral density estimate we use the usual periodogram with and without taper, where we apply splitcosine-tapering (Priestley 1981). The parameter $0<$ $\beta \leq 0.5$ corresponds to the part of the time series sequence that is weighted down at the beginning and the end of the sequence.

- Estimators are investigated, which are calculated from SLEX periodograms. A taper effect is implicitly given here by the two window functions $\vartheta^{+}$and $\vartheta^{-}$. We define the parameter $\beta$ as $\beta=2 \varepsilon / m$ with $m=N / 2+\varepsilon$.

- For smoothing we use a Daniell estimator (Koopmans 1974), which is a simple smoothed periodogram with equal weights on all frequencies in a window of width $2 d+1, d \in \mathbb{N}$. 
- As an alternative we use estimators calculated by splitting the given time window into eight segments and averaging over the corresponding periodograms.

- In addition, we use a spectral density estimator from an autoregressive (AR) model of order $p$ minimizing the AIC (Choi 1992).

Those three processes are chosen out of a great amount of processes that show the smallest difference of chattering and non-chattering segments but are nevertheless classified as chattering process by an expert. Out of each of the three processes 50 steady and 50 chattering segments are chosen.

Each of the chatter-free segments is compared to each other and to all chattering sequences within each process by calculating the divergence measures between the spectral density estimates of the segments. Overall, there are $3 \times 1225=3675$ comparisons of two chatter-free processes and $3 \times 2500=7500$ comparisons between one chatterfree and one chattering segment. We investigate how many changes can be detected with the help of a limit for the divergence measures. This limit is fixed so that only a certain percentage of comparisons of two calm process parts results in the detection of a difference. The limit will be fixed here to the empirical $90 \%(95 \%, 99 \%)$ quantile of the values of the divergence measures from the 3675 comparisons of two chatter-free segments. Every value exceeding this limit shows by definition a discrepancy between the spectra of two time series segments. The fraction of values of the divergence measures in the 7500 comparisons of differently classified segments which exceed the limit serves as a criterion for the comparison of the divergence measures. This fraction can be interpreted as the sensitivity, which is the estimated probability that a difference is detected, if a chatter-free segment is compared to a chattering segment. In the same way the fixed $90 \%(95 \%, 99 \%)$ can be interpreted as the specificity which is the estimated probability that no difference is noticed, if two chatter-free segments are compared.

Figure 2 shows the performance of the divergence measures by giving the sensitivities depending on the fixed specificities for the case of SLEX periodograms which overlap with $\beta=0.5$. As can be seen, the Itakura-Saïto divergence outperforms the other measures. Tables 1-5 in Appendix B show the sensitivities calculated from the data as resulting from the complete study. When using just the periodogram or the SLEX periodogram without any kind of smoothing to decrease the variance other measures are better. But the Itakura-Saïto divergence shows the best performance if we use estimators with a reasonably small variance by some kind of sensible smoothing in frequency or time direction.

\section{CONTROL CHARTS}

In a control chart the asymptotic uncorrelated SLEX periodogram values will be smoothed over time with an expo-

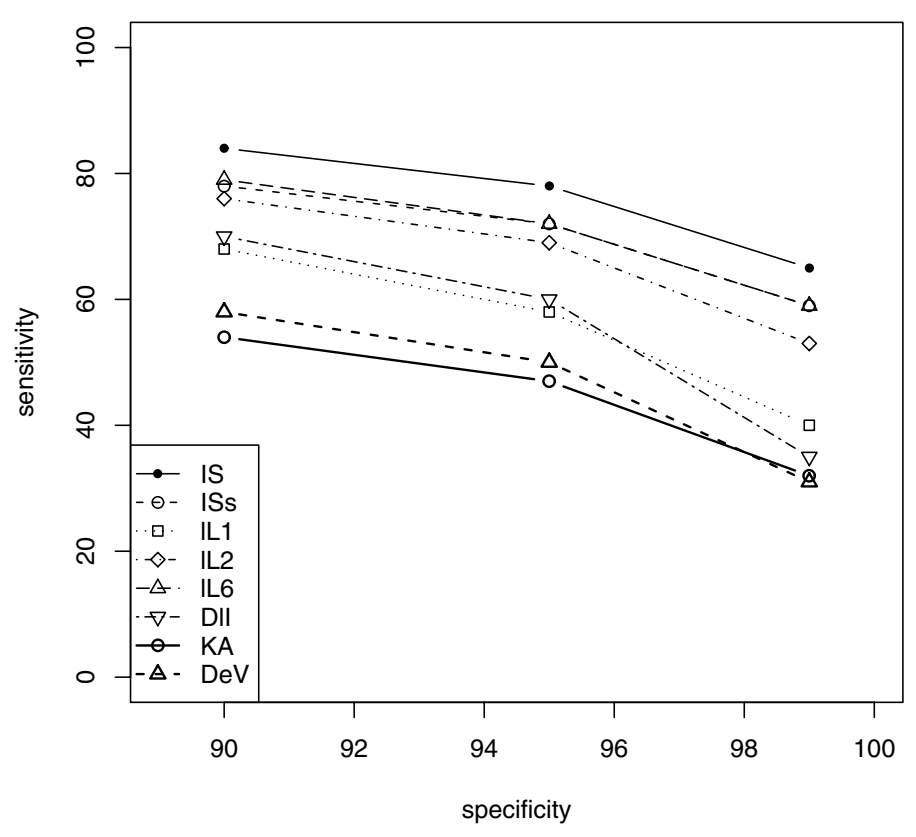

Figure 2. Sensitivities in \% Against the Specificity in \% (Fixed at 90, 95 and 99\%) of Different Spectral Divergence Measures. The Spectral Density Is Estimated by SLEX Periodograms with $\beta=0.5$ and Smoothed Over Time.

nentially weighted moving average

$$
Z_{j, k}=\lambda \hat{f}_{k}\left(\omega_{j}\right)+(1-\lambda) Z_{j, k-1},
$$

for all $j \in\{1,2, \ldots, N / 2\}$ and $0<\lambda \leq 1$. This is a compromise between a fast adaptation to the changes in the spectrum and a good estimation of the spectral density if the process is stationary.

Theorem 2. Let $\left\{X_{t}\right\}_{t} \in \mathbb{Z}$ be a stationary process with spectral density $f$. Furthermore let the assumptions of Theorem 1 be valid.

Let $I_{k}(\omega)$ be the SLEX periodogram on the $k$-th segment with length $N$. Let a recursive estimation function for $f(\omega)$ be defined as

$$
Z_{j, k}=\lambda I_{k}\left(\omega_{j}\right)+(1-\lambda) Z_{j, k-1},
$$

where $\lambda \in(0,1), k \in \mathbb{N}$ and $\hat{f}_{0}(\omega)=0$. Then,

$$
\mathrm{E}\left(Z_{j, k}\right) \underset{N \rightarrow \infty}{\longrightarrow} f\left(\omega_{j}\right) \text { for all } k,
$$

and

$$
\operatorname{Var}\left(Z_{j, k}\right) \underset{N \rightarrow \infty}{\stackrel{k \rightarrow \infty}{\longrightarrow}} \frac{\lambda}{2-\lambda} f^{2}\left(\omega_{j}\right) .
$$

The proof is given in Appendix A.

Thus, $Z_{j, k}$, the asymptotically unbiased estimator of the spectral density, has small variance when $\lambda$ is small, because the values that are summed up are approximately 
uncorrelated. The divergence of this smoothed time series $\left(Z_{1, k}, \ldots, Z_{N / 2, k}\right)$ of the periodogram values from the reference periodogram denoted by $\left(\hat{\mu}_{1, k}, \ldots, \hat{\mu}_{N / 2, k}\right)$ is measured by the Itakura-Saito divergence. An alarm is given as soon as this divergence is too large or $d_{\mathrm{IS}}>h, h \geq 0$, with

$$
d_{\mathrm{IS}, k}\left(Z_{k}, \hat{\mu}_{k}\right)=\sum_{j=1}^{N / 2}\left(\frac{Z_{j, k}}{\hat{\mu}_{j, k}}-\ln \frac{Z_{j, k}}{\hat{\mu}_{j, k}}-1\right), \quad k \in \mathbb{N} .
$$

The smoothing in time solves two problems in one step. Firstly, smoothing is needed to assure the good performance of the distance measure. Secondly, smoothing counteracts variability in the spectrum that would lead to false alarms.

Moreover, to avoid that small irrelevant changes trigger an alarm, the reference values $\left(\hat{\mu}_{1, k}, \ldots, \hat{\mu}_{N / 2, k}\right)$ are constructed as slowly adapting exponentially weighted moving averages of the periodogram values with

$$
\hat{\mu}_{j, k}=\nu \hat{f}_{k-a}\left(\omega_{j}\right)+(1-\nu) \hat{\mu}_{j, k-1},
$$

for all $j \in\{1,2, \ldots, N / 2\}$, and $0<\nu \ll \lambda \leq 1$. The reference series $\left\{\hat{\mu}_{j, k}\right\}_{k}$ adapts itself more slowly to the changes in the spectrum than the test series $\left\{Z_{j, k}\right\}_{k}$ because a delay value $a \in\{1,2, \ldots\}$ is introduced and $\nu$ is smaller than $\lambda$. Here, $\left(Z_{1,0}, \ldots, Z_{N / 2,0}\right)$ and $\left(\hat{\mu}_{1,0}, \ldots, \hat{\mu}_{N / 2,0}\right)$ are starting vectors, and $\hat{f}_{-a}\left(\omega_{j}\right), \hat{f}_{-a+1}\left(\omega_{j}\right), \ldots, \hat{f}_{0}\left(\omega_{j}\right)$ are spectral density estimates. These could be calculated with the help of the SLEX periodogram e.g. out of the idle running phase in the process.

This general setting of a control chart can be specified as follows: For $\nu=1$ and $\lambda=1$ we construct a delayed moving window technique. For $\lambda=1$ only the recent spectrum is compared to the past. Furthermore, we can allow $\nu=0$ (also $a=0$ ) for the comparison with a fixed reference signal $\left(\hat{\mu}_{1}, \ldots, \hat{\mu}_{N / 2}\right)$. From Section 3 we know that the ItakuraSaïto divergence only works well, if a variance-reduced spectral representation is used. If there is no smoothing over the different time points we need to smooth the SLEX periodograms over the frequencies.

\section{APPLICATION}

Even in very stable drilling processes the spot drilling causes a change in the spectrum of the structure-borne sound signal. This is why the control charts start some seconds after spot drilling and why the burn in phase is used as reference signal. A control chart can be applied for the detection of chatter during or shortly after spot drilling (Busch 2006). It uses the spectrum of the idle running phase as reference values $\left(\hat{\mu}_{1, k}, \ldots, \hat{\mu}_{N / 2, k}\right)$. This is the special case of the above control chart with $\nu=0, a=0$.

The online control of the drilling phase after a chatter free state is carried out by the control chart in its general setting. The choice of the limit $h$ and of other control chart

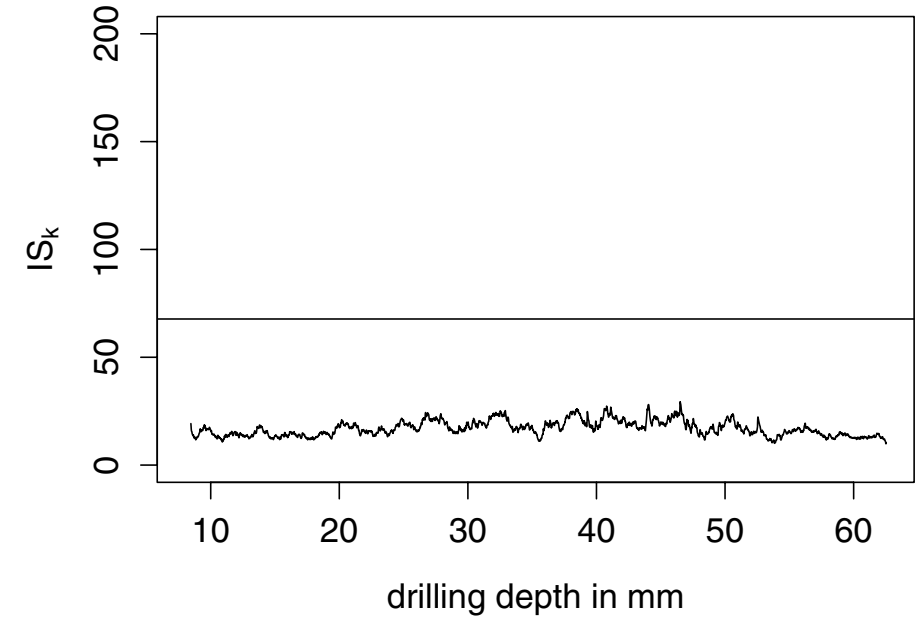

Figure 3. Control Chart for Structure Borne Sound in a Process Without Chatter.

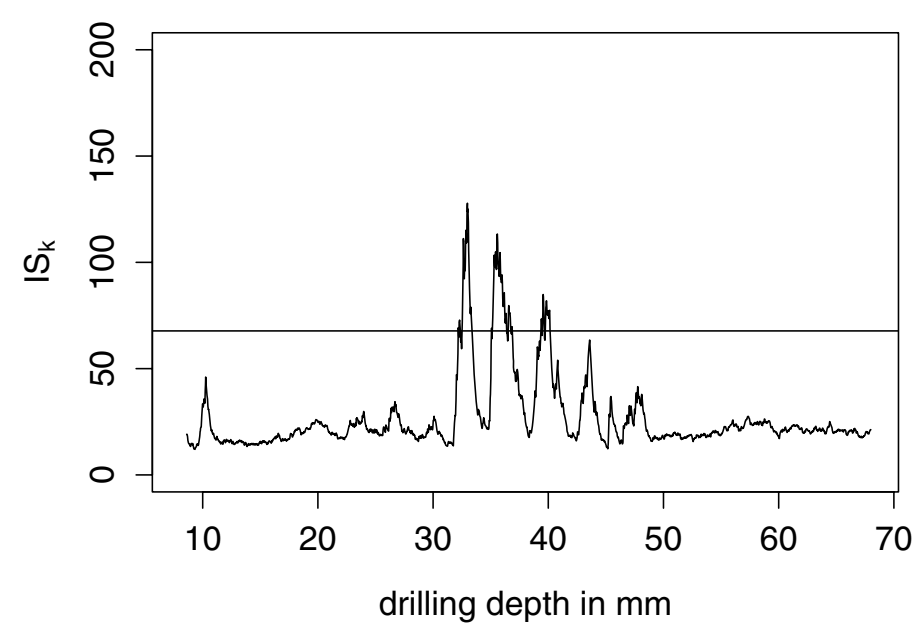

Figure 4. Control Chart for Structure Borne Sound Applied to a Process Without Noticed Chatter but with Strong Disturbances.

parameters is based on the investigation of 24 chatter-free drilling processes. The parameters of the control chart are fixed as $N=256, a=3, \lambda=0.05$, and such that the SLEX windows overlap by 128 values. Furthermore, the parameter $\nu=0.0005$ is chosen such that in all chatter-free cases the statistic is at a steady level. The control limit is fixed to $h \approx 67.75$ such that for the 24 chatter free processes $98 \%$ of the control chart values lie below $h$. Figures $3,4,5,6$ and 7 show how other process examples are evaluated by the chart. The control chart statistic reacts sensibly to turbulent phases resulting in high amplitudes. In general, with the given control limits no alarm is given in calm processes as seen in Figure 3. Phases, where chatter vibration occurs are detected fast as can be seen in Figure 5,6 and 7, but there 


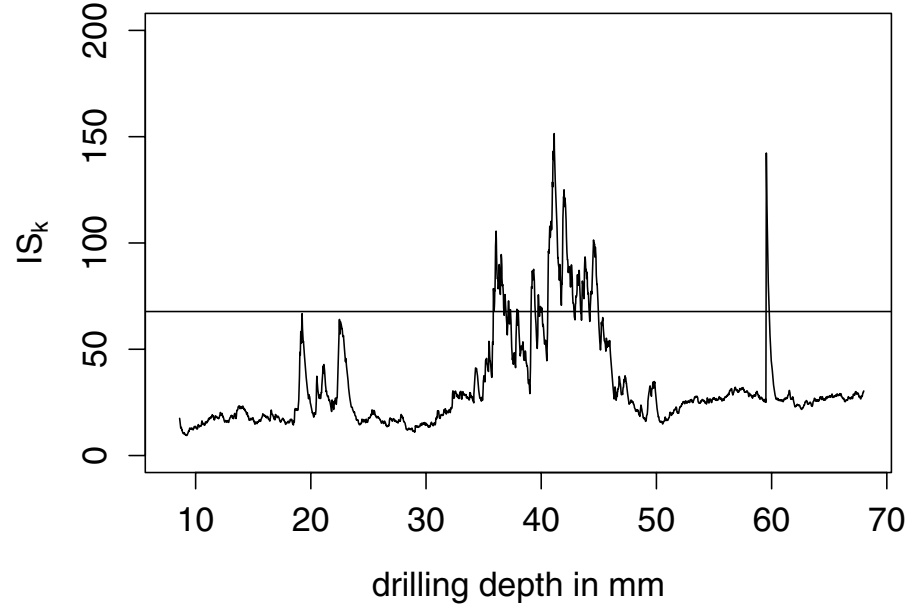

Figure 5. Control Chart for Structure Borne Sound Applied to a Process with Slight Chatter in the Second Half.

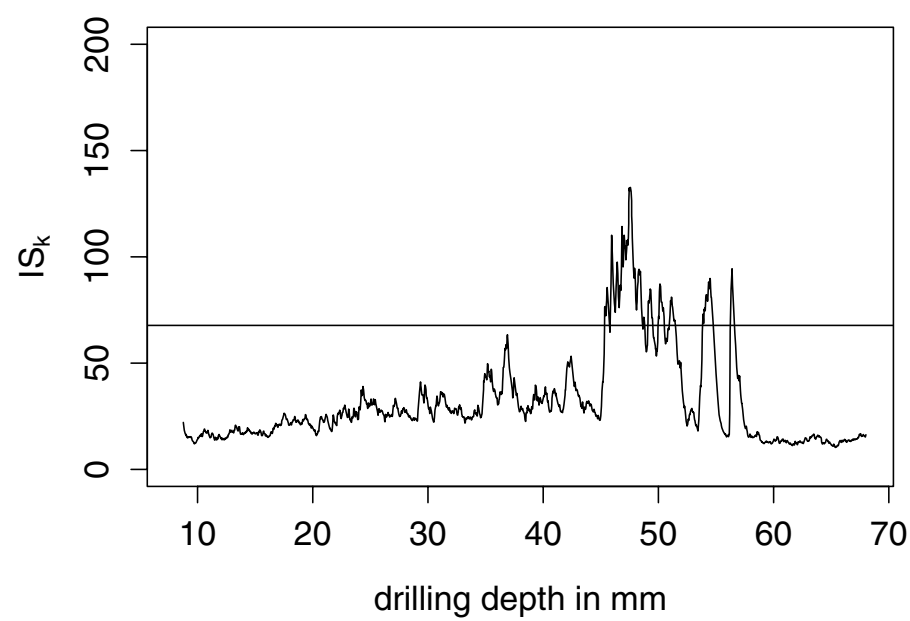

Figure 6. Control Chart for Structure Borne Sound Applied to a Process with Slight Chatter in the Second Half.

is also one false alarm sound in Figure 4. One can however conjecture that the expert missed the chatter vibration in this process.

\section{CONCLUSIONS}

Summarizing, we can say that the chatter vibration in single-lip deep-hole drilling expresses itself in an increase of power in some frequency bands of the spectrum relative to the steady situation. By means of an empirical comparison of diverse spectral divergence measures we found out that the Itakura-Saïto divergence is the most separating measure. Based on this measure, we have developed a control chart which can detect changes in the spectrum and which is able to distinguish chatter from calm process parts. This control chart uses exponentially weighted averages of the SLEX pe-

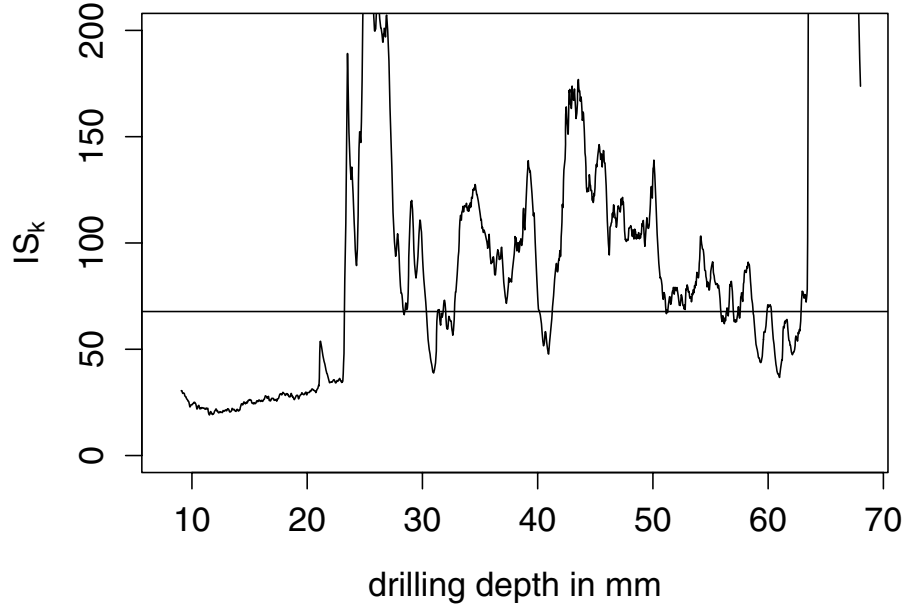

Figure 7. Control Chart for Structure Borne Sound Applied to a Process with Strong Chatter After $20 \mathrm{~mm}$.

riodogram values, which are asymptotically uncorrelated in adjacent time windows.

\section{ACKNOWLEDGEMENT}

This work was partially supported by the Graduate School of Production Engineering and Logistics, Universität Dortmund.

\section{APPENDIX A: PROOFS}

\section{Proof of Theorem 1.}

The SLEX periodograms are given as

$$
I_{k}(\omega)=\frac{1}{N}\left|\sum_{t=-m+1}^{m} \varphi_{\omega}^{*}(t) X_{t+m+(k-1) N}\right|^{2},
$$

with

$$
\varphi_{\omega}(t)=\vartheta^{+}(t) e^{i t \omega}+\vartheta^{-}(t) e^{-i t \omega}
$$

and $\vartheta^{+}(t), \vartheta^{-}(t)$ the corresponding real window functions with support $\{-m+1, \ldots, m\}$.

We only prove $\operatorname{Cov}\left(I_{k}\left(\omega_{j}\right), I_{h}\left(\omega_{j}\right)\right)=\mathrm{O}\left(1 / N^{2}\right)$ for all $k$ with $h=k+1$ and all $j$. The proof is easily generalized to cases where $h>k+1$.

From Brillinger (1981), page 21 we get

$$
\begin{aligned}
\mathrm{E}\left(X_{1} X_{2} X_{3} X_{4}\right)= & \mathrm{E}\left(X_{1} X_{2}\right) \mathrm{E}\left(X_{3} X_{4}\right) \\
& +\mathrm{E}\left(X_{1} X_{3}\right) \mathrm{E}\left(X_{2} X_{4}\right) \\
& +\mathrm{E}\left(X_{1} X_{4}\right) \mathrm{E}\left(X_{2} X_{3}\right) .
\end{aligned}
$$


This implies

(3)

$$
\begin{aligned}
\mathrm{E} & \left(I_{k}(\omega) I_{k+1}(\omega)\right) \\
= & \left(\frac{1}{N} \sum_{t=-m+1}^{m} \sum_{s=-m+1}^{m} \varphi_{\omega}^{*}(t) \varphi_{\omega}(s) \int_{-\pi}^{\pi} f(\lambda) e^{i(t-s) \lambda} d \lambda\right)^{2} \\
& +\left|\frac{1}{N} \sum_{t=-m+1}^{m} \sum_{s=-m+1}^{m} \varphi_{\omega}(t) \varphi_{\omega}(s) \int_{-\pi}^{\pi} f(\lambda) e^{i(t-s+N) \lambda} d \lambda\right|^{2} \\
& +\left|\frac{1}{N} \sum_{t=-m+1}^{m} \sum_{s=-m+1}^{m} \varphi_{\omega}^{*}(t) \varphi_{\omega}(s) \int_{-\pi}^{\pi} f(\lambda) e^{i(t-s+N) \lambda} d \lambda\right|^{2} .
\end{aligned}
$$

which implies

$$
\begin{aligned}
& \left|\frac{1}{N} \int_{-\pi}^{\pi} \hat{\vartheta}^{+}(-\omega+\lambda) \hat{\vartheta}^{-}(\omega-\lambda) f(\lambda) e^{i N \lambda} d \lambda\right| \\
& \quad \leq\left|\frac{1}{N} \int_{-\pi}^{\pi} \hat{\vartheta}^{+}(-\omega+\lambda) \hat{\vartheta}^{-}(\omega-\lambda) f(\lambda) d \lambda\right| \\
& \quad=\mathrm{O}(1) .
\end{aligned}
$$

In the same manner we get

$$
\left|\frac{1}{N} \int_{-\pi}^{\pi} \hat{\vartheta}^{+}(-\omega-\lambda) \hat{\vartheta}^{-}(\omega+\lambda) f(\lambda) e^{i N \lambda} d \lambda\right|=\mathrm{O}(1)
$$

Using Theorem 2 in Ombao et al. (2001), the first summand in (3) is equal to

$$
\mathrm{E}\left(I_{k}(\omega)\right)^{2}=(f(\omega)+\mathrm{O}(1 / N)+\mathrm{o}(1 / N))^{2} .
$$

For the second summand in (3) one gets

$$
\begin{aligned}
& \mid \frac{1}{N} \int_{-\pi}^{\pi} \hat{\vartheta}^{+}(-\omega-\lambda) \hat{\vartheta}^{+}(-\omega+\lambda) f(\lambda) e^{i N \lambda} d \lambda \\
& \quad+\frac{1}{N} \int_{-\pi}^{\pi} \hat{\vartheta}^{-}(\omega-\lambda) \hat{\vartheta}^{-}(\omega+\lambda) f(\lambda) e^{i N \lambda} d \lambda \\
& \quad+\frac{1}{N} \int_{-\pi}^{\pi} \hat{\vartheta}^{+}(-\omega-\lambda) \hat{\vartheta}^{-}(\omega+\lambda) f(\lambda) e^{i N \lambda} d \lambda \\
& \quad+\left.\frac{1}{N} \int_{-\pi}^{\pi} \hat{\vartheta}^{+}(-\omega+\lambda) \hat{\vartheta}^{-}(\omega-\lambda) f(\lambda) e^{i N \lambda} d \lambda\right|^{2}
\end{aligned}
$$

by exchange of integration and summation with the help of the Fourier transform $\hat{\vartheta}^{+}$and $\hat{\vartheta}^{-}$of $\vartheta^{+}$and $\vartheta^{-}$and by some numerical manipulations. Because $\hat{\vartheta}^{+}$and $\hat{\vartheta}^{-}$are highly concentrated at 0 , we get for $\omega \neq 0$

$$
\frac{1}{N} \int_{-\pi}^{\pi} \hat{\vartheta}^{+}(-\omega-\lambda) \hat{\vartheta}^{+}(-\omega+\lambda) f(\lambda) e^{i N \lambda} d \lambda=\mathrm{o}(1 / N)
$$

and

$$
\frac{1}{N} \int_{-\pi}^{\pi} \hat{\vartheta}^{-}(\omega-\lambda) \hat{\vartheta}^{-}(\omega+\lambda) f(\lambda) e^{i N \lambda} d \lambda=\mathrm{o}(1 / N) .
$$

One can show that

$$
\begin{gathered}
\operatorname{Cov}\left(\sum_{t} \vartheta^{+}(t) X_{t} e^{-i t \lambda}, \sum_{t} \vartheta^{-}(t) X_{t} e^{-i t \lambda}\right) \\
=\int_{-\pi}^{\pi} \hat{\vartheta}^{+}(\lambda-\nu) \hat{\vartheta}^{-}(\nu-\lambda) f(\nu) d \nu
\end{gathered}
$$

Theorem 4.3.2 in Brillinger (1981) yields

$$
\begin{gathered}
\operatorname{Cov}\left(\sum_{t} \vartheta^{+}(t) X_{t} e^{-i t \lambda}, \sum_{t} \vartheta^{-}(t) X_{t} e^{-i t \lambda}\right) \\
=\sum_{t} \vartheta^{+}(t) \vartheta^{-}(t) f(\lambda)+\mathrm{O}(1)=\mathrm{O}(1) .
\end{gathered}
$$

Therefore, the second summand in (3) is equal to $(2 \mathrm{o}(1 / N)+$ $2 \mathrm{O}(1 / N))^{2}$.

Under the assumption that $f(\omega)=f(-\omega)$ is sufficiently smooth, and because the Fourier transform $\Phi_{\omega}$ of $\varphi_{\omega}$ is highly concentrated at $\omega$ and $-\omega$, the third summand in (3) can be written as

$\left.\left.f(\omega)^{2}\left|\frac{1}{N} \int_{-\pi}^{\pi}\right| \Phi_{\omega}(\lambda)\right|^{2} e^{i N \lambda} d \lambda\right|^{2}=\frac{1}{N^{2}}\left(\sum_{t} \varphi_{\omega}^{*}(t) \varphi_{\omega}(t+N)\right)^{2}$.

The last equation is given in Grooves and Hannan (1968, p. 138), where the sum is over all $t$ for which simultaneously hold $t \in\{-m+1, \ldots, m\}$ and $t+N \in\{-m+1, \ldots, m\}$. Because $\sum_{t} \varphi_{\omega}^{*}(t) \varphi_{\omega}(t+N)=0$ and because of the orthogonality of the SLEX function the third summand in (3) is equal to 0 .

$$
\begin{aligned}
& \text { Summarizing, we get } \\
& \begin{aligned}
\operatorname{Cov}\left(I_{k}(\omega), I_{k+1}(\omega)\right) \\
=\mathrm{E}\left(I_{k}(\omega) I_{k+1}(\omega)\right)-\mathrm{E}\left(I_{k}(\omega)\right) \mathrm{E}\left(I_{k+1}(\omega)\right) \\
=\mathrm{E}\left(I_{k}(\omega)\right)^{2}+(2 \mathrm{O}(1 / N)+2 \mathrm{O}(1 / N))^{2}-\mathrm{E}\left(I_{k}(\omega)\right)^{2} \\
=\mathrm{O}\left(1 / N^{2}\right) \quad \forall k .
\end{aligned}
\end{aligned}
$$

The second statement

$$
\operatorname{Cov}\left(I_{k}\left(\omega_{j}\right), I_{k}\left(\omega_{i}\right)\right)=\mathrm{O}(1 / N) \quad \forall i \neq j
$$

can be proven as in Theorem 5.2.4 in Brillinger (1981).

\section{Proof of Theorem 2.}

From Theorem 2 in Ombao et al. (2001) we have

$$
\begin{aligned}
\mathrm{E}\left(I_{k}(\omega)\right) & =f(\omega)+\mathrm{O}(1 / N)+\mathrm{o}(1 / N), \\
\operatorname{Var}\left(I_{k}(\omega)\right) & =f^{2}(\omega)+\mathrm{O}(1 / N),
\end{aligned}
$$

such that

$$
\mathrm{E}\left(Z_{j, k}\right)=f(\omega)+\mathrm{O}(1 / N)+\mathrm{o}(1 / N)
$$


and

$$
\begin{aligned}
\operatorname{Var} & \left.Z_{j, k}\right) \\
= & \operatorname{Var}\left(\lambda \sum_{\nu=1}^{k}(1-\lambda)^{k-\nu} I_{\nu}(\omega)\right) \\
= & \lambda^{2} \sum_{\nu=1}^{k}(1-\lambda)^{2(k-\nu)} \operatorname{Var}\left(I_{\nu}(\omega)\right) \\
& +\lambda^{2} \sum_{\nu \neq l}(1-\lambda)^{k-\nu}(1-\lambda)^{k-l} \operatorname{Cov}\left(I_{\nu}(\omega), I_{l}(\omega)\right) \\
= & \left(f^{2}(\omega)+\mathrm{O}(1 / N)\right) \lambda^{2} \sum_{\nu=1}^{k}(1-\lambda)^{2(k-\nu)} \\
& +\mathrm{O}\left(1 / N^{2}\right)\left(\lambda \sum_{\nu=1}^{k}(1-\lambda)^{k-\nu}\right)^{2} \\
& -\mathrm{O}\left(1 / N^{2}\right) \lambda^{2} \sum_{\nu=1}^{k}(1-\lambda)^{2(k-\nu)} \\
= & \left(f^{2}(\omega)+\mathrm{O}(1 / N)-\mathrm{O}\left(1 / N^{2}\right)\right) \frac{\lambda}{2-\lambda}\left(1-(1-\lambda)^{2 k}\right) \\
& +\mathrm{O}\left(1 / N^{2}\right)\left(1-(1-\lambda)^{k}\right)^{2} \\
\underset{k \rightarrow \infty}{\longrightarrow} & \frac{\lambda}{2-\lambda}\left(f^{2}(\omega)+\mathrm{O}(1 / N)-\mathrm{O}\left(1 / N^{2}\right)\right)+\mathrm{O}\left(1 / N^{2}\right) .
\end{aligned}
$$

\section{APPENDIX B: TABLES WITH SENSITIVITIES OF DIVERGENCE MEASURES}

Table 1. Sensitivities in \% with 90, 95 and 99\% Specificity (sp.) of Different Spectral Divergence Measures and Different

Estimators (Smoothing Coefficient (d), Taper Coefficient

$(\beta))$. The Highest Sensitivity Denoting the Best Performing Measure Is Underlined

\begin{tabular}{|c|c|c||c|c|r|r|r|r|r|r|}
\hline sp. & $\beta$ & $d$ & IS & ISs & lL1 & lL2 & lL6 & DlI & KA & DeV \\
\hline \hline 90 & 0 & 0 & 16 & 14 & 46 & 47 & 16 & 48 & $\underline{61}$ & 58 \\
\hline 95 & 0 & 0 & 7 & 7 & 34 & 35 & 7 & 36 & $\underline{54}$ & 50 \\
\hline 99 & 0 & 0 & 1 & 1 & 18 & 19 & 1 & 20 & $\underline{34}$ & 31 \\
\hline \hline 90 & 0.1 & 0 & 43 & 22 & 51 & 55 & 27 & $\underline{62}$ & 60 & 58 \\
\hline 95 & 0.1 & 0 & 25 & 9 & 39 & 45 & 11 & 49 & $\underline{52}$ & 50 \\
\hline 99 & 0.1 & 0 & 3 & 2 & 21 & 27 & 3 & 29 & $\underline{32}$ & 31 \\
\hline \hline 90 & 0 & 4 & $\underline{89}$ & 84 & 70 & 81 & 88 & 64 & 61 & 58 \\
\hline 95 & 0 & 4 & $\underline{83}$ & 77 & 57 & 73 & 81 & 52 & 53 & 50 \\
\hline 99 & 0 & 4 & 65 & 60 & 35 & 54 & $\underline{67}$ & 30 & 32 & 31 \\
\hline \hline 90 & 0.1 & 4 & $\underline{93}$ & 90 & 75 & 88 & 92 & 69 & 60 & 58 \\
\hline 95 & 0.1 & 4 & $\underline{89}$ & 85 & 64 & 80 & $\underline{89}$ & 59 & 52 & 50 \\
\hline 99 & 0.1 & 4 & $\underline{76}$ & 71 & 40 & 61 & 75 & 34 & 31 & 31 \\
\hline \hline 90 & 0.2 & 4 & $\underline{92}$ & 88 & 72 & 85 & 91 & 66 & 56 & 58 \\
\hline 95 & 0.2 & 4 & $\underline{87}$ & 83 & 61 & 77 & $\underline{87}$ & 55 & 48 & 50 \\
\hline 99 & 0.2 & 4 & $\underline{73}$ & 68 & 36 & 59 & 72 & 28 & 30 & 31 \\
\hline \hline 90 & 0.1 & 5 & $\underline{93}$ & 90 & 74 & 87 & 92 & 71 & 60 & 58 \\
\hline 95 & 0.1 & 5 & $\underline{89}$ & 84 & 64 & 80 & $\underline{89}$ & 59 & 52 & 50 \\
\hline 99 & 0.1 & 5 & $\underline{76}$ & 69 & 40 & 62 & 74 & 35 & 31 & 31 \\
\hline
\end{tabular}

Table 2. Sensitivities in \% with 90, 95 and 99\% Specificity (sp.) of Different Spectral Divergence Measures. The Spectral Density Is Estimated by Periodograms in Smaller Parts of Time Series which Are Averaged Over Time. The Highest Sensitivity Denoting the Best Performing Measure Is Underlined

\begin{tabular}{|c|c||c|c|c|c|c|c|c|c|}
\hline sp. & $\beta$ & IS & ISs & lL1 & lL2 & lL6 & DlI & KA & DeV \\
\hline \hline 90 & 0.1 & $\underline{86}$ & 81 & 67 & 79 & 83 & 70 & 58 & 58 \\
\hline 95 & 0.1 & $\underline{82}$ & 75 & 56 & 71 & 78 & 59 & 54 & 50 \\
\hline 99 & 0.1 & $\underline{72}$ & 62 & 40 & 57 & 65 & 37 & 35 & 31 \\
\hline \hline 90 & 0.2 & $\underline{88}$ & 83 & 68 & 81 & 86 & 68 & 58 & 58 \\
\hline 95 & 0.2 & $\underline{83}$ & 78 & 58 & 74 & 80 & 56 & 52 & 50 \\
\hline 99 & 0.2 & $\underline{74}$ & 67 & 40 & 62 & 71 & 32 & 34 & 31 \\
\hline \hline 90 & 0.3 & $\underline{86}$ & 81 & 67 & 79 & 83 & 65 & 57 & 58 \\
\hline 95 & 0.3 & $\underline{81}$ & 75 & 57 & 72 & 78 & 54 & 48 & 50 \\
\hline 99 & 0.3 & $\underline{71}$ & 64 & 38 & 57 & $\underline{71}$ & 30 & 33 & 31 \\
\hline
\end{tabular}

Table 3. Sensitivities in \% with 90, 95 and 99\% Specificity (sp.) of Different Spectral Divergence Measures. The Spectral Density Is Estimated by Fitting an AR Model and Using Its Spectral Density. The Highest Sensitivity Denoting the Best Performing Measure Is Underlined

\begin{tabular}{|c||c|c|c|c|c|c|c|c|}
\hline sp. & IS & ISs & lL1 & lL2 & lL6 & DlI & KA & DeV \\
\hline \hline 90 & $\underline{85}$ & 80 & 72 & 79 & 81 & 65 & 61 & 58 \\
\hline 95 & $\underline{79}$ & 73 & 60 & 72 & 74 & 54 & 54 & 50 \\
\hline 99 & $\underline{65}$ & 58 & 33 & 54 & 64 & 30 & 34 & 31 \\
\hline
\end{tabular}

Table 4. Sensitivities in \% with 90, 95 and 99\% Specificity (sp.) of Different Spectral Divergence Measures. The Spectral Density Is Estimated by SLEX Periodograms Smoothed Over Frequencies. The Highest Sensitivity Denoting the Best Performing Measure Is Underlined

\begin{tabular}{|c|c|r||r|r|r|r|r|r|r|r|}
\hline sp. & $\beta$ & $d$ & IS & ISs & lL1 & lL2 & lL6 & DlI & KA & DeV \\
\hline \hline 90 & $1 / 8$ & 0 & 43 & 30 & 59 & 62 & 36 & $\underline{64}$ & 43 & 58 \\
\hline 95 & $1 / 8$ & 0 & 25 & 16 & 48 & $\underline{51}$ & 22 & $\underline{51}$ & 38 & 50 \\
\hline 99 & $1 / 8$ & 0 & 6 & 4 & 29 & $\underline{32}$ & 10 & 26 & 30 & 31 \\
\hline \hline 90 & $1 / 8$ & 4 & $\underline{91}$ & 87 & 74 & 84 & 89 & 68 & 44 & 58 \\
\hline 95 & $1 / 8$ & 4 & $\underline{86}$ & 82 & 66 & 78 & 85 & 56 & 38 & 50 \\
\hline 99 & $1 / 8$ & 4 & $\underline{74}$ & 71 & 46 & 64 & 73 & 33 & 30 & 31 \\
\hline \hline 90 & $1 / 2$ & 4 & $\underline{94}$ & 89 & 76 & 86 & 91 & 72 & 52 & 58 \\
\hline 95 & $1 / 2$ & 4 & $\underline{89}$ & 83 & 66 & 80 & 87 & 59 & 43 & 50 \\
\hline 99 & $1 / 2$ & 4 & $\underline{72}$ & 68 & 37 & 58 & 71 & 19 & 31 & 31 \\
\hline \hline 90 & $1 / 8$ & 5 & $\underline{91}$ & 86 & 74 & 83 & 89 & 68 & 44 & 58 \\
\hline 95 & $1 / 8$ & 5 & $\underline{85}$ & 81 & 65 & 78 & $\underline{85}$ & 56 & 38 & 50 \\
\hline 99 & $1 / 8$ & 5 & $\underline{73}$ & 70 & 45 & 64 & 71 & 34 & 30 & 31 \\
\hline
\end{tabular}


Table 5. Sensitivities in \% with 90, 95 and 99\% Specificity (sp.) of Different Spectral Divergence Measures. The Spectral Density Is Estimated by SLEX Periodograms Smoothed Over Time. The Highest Sensitivity Denoting the Best Performing Measure Is Underlined

\begin{tabular}{|c|c||c|c|c|c|c|c|c|c|}
\hline sp. & $\beta$ & IS & ISs & lL1 & lL2 & lL6 & DlI & KA & DeV \\
\hline \hline 90 & $1 / 8$ & $\underline{75}$ & 66 & 61 & 64 & 69 & 69 & 52 & 58 \\
\hline 95 & $1 / 8$ & $\underline{66}$ & 50 & 50 & 56 & 60 & 57 & 44 & 50 \\
\hline 99 & $1 / 8$ & $\underline{49}$ & 40 & 30 & 38 & 44 & 33 & 30 & 31 \\
\hline \hline 90 & $1 / 4$ & $\underline{80}$ & 72 & 64 & 70 & 74 & 72 & 50 & 58 \\
\hline 95 & $1 / 4$ & $\underline{74}$ & 66 & 55 & 64 & 67 & 62 & 42 & 50 \\
\hline 99 & $1 / 4$ & $\underline{58}$ & 51 & 37 & 48 & 55 & 41 & 30 & 31 \\
\hline \hline 90 & $1 / 2$ & $\underline{84}$ & 78 & 68 & 76 & 79 & 70 & 54 & 58 \\
\hline 95 & $1 / 2$ & $\underline{78}$ & 72 & 58 & 69 & 72 & 60 & 47 & 50 \\
\hline 99 & $1 / 2$ & $\underline{65}$ & 59 & 40 & 53 & 59 & 35 & 32 & 31 \\
\hline
\end{tabular}

\section{Received 7 February 2008}

\section{REFERENCES}

Basseville, M. (1989). Distance Measures for Signal Processing and Pattern Recognition. Signal Processing 18 349-369. MR1028051

Basseville, M. and Nikiforov, I. V. (1993). Detection of Abrupt Changes: Theory and Application. Prentice Hall, Englewood Cliffs, New Jersey. MR1210954

Brillinger, D. R. (1981). Time Series: Data Analysis and Theory. Holden Day, San Francisco. MR0595684

Busch, A. S. (2006). Statistische Analyse und Rattererkennung beim Einlippen-Tiefbohren. Dissertation, Universität Dortmund, Fachbereich Statistik, URL: http://hdl.handle.net/2003/22278.

Carmona, R., Hwang, W.-L. and Torrésani, B. (1998). Practical Time-Frequency Analysis - Gabor and Wavelet Transforms with an Implementation in S. Academic Press, San Diego. MR1667366

CHOI, B. (1992). ARMA Model Identification. Springer-Verlag, New York. MR1220907

Eichler, M. (2007). Testing Nonparametric and Semiparametric Hypotheses in Vector Stationary Processes. Journal of Multivariate Analysis, in print, doi:10.1016/j.jmva.2007.06.003

Gray, A. H. and Markel, J. D. (1976). Distance Measures for Speech Processing. IEEE Transactions on Acoustics, Speech, and Signal Processing 24(5) 380-391.

Groves, G. W. and Hannan, E. J. (1968). Time Series Regression of Sea Level on Weather. Reviews of Geophysics 6(2) 129-174.

Koopmans, L. H. (1974). The Spectral Analysis of Time Series. Academic Press, New York. MR0348953
Ligges, U., Weins, C. and Hasse-Becker, P. (2002). Detection of Locally Stationary Segments in Time Series-Algorithms and Applications. Technical Report 11/2002, SFB 475, Universität Dortmund. MR1986555

Lowry, C. A., Woodall, W. H., Champ, C. W. and Rigdon, S. E. (1992). A Multivariate Exponentially Weighted Moving Average Control Chart. Technometrics 34(1) 46-53.

Ombao, H., Heo, J. and Stoffer, D. (2004). Online Analysis of Seismic Signals. In Time Series Analysis and Applications to Geophysical Systems, D. R. Brillinger, E. A. Robinson, and F. P. Schoenberg, eds. Springer-Verlag, New York, pp. 53-71. MR2111481

Ombao, H. C., Raz, J. A., von Sachs, R. and Malow, B. A. (2001). Automatic Statistical Analysis of Bivariate Nonstationary Time Series. Journal of the American Statistical Association, Theory and Methods 96(454) 543-560. MR1946424

Page, E. S. (1961). Cumulative Sum Charts. Technometrics 3(1) 1-9. MR0119344

PicARD, D. (1985). Testing and Estimating Change-Points in Time Series. Advances in Applied Probability 17 841-867. MR0809433

Priestley, M. B. (1981). Spectral Analysis and Time Series-1: Univariate Series. Academic Press, London. MR0628735

Priestley, M. B. (1988). Non-Linear and Non-Stationary Time Series Analysis. Academic Press, London.

Subba RaO, T. (1981). A Cumulative Sum Test for Detecting Change in Time Series. International Journal of Control 34(2) 285-293. MR0631818

Weinert, K., Peters, C. and Mehnen, J. (2002). Rechnerbasierte Prozess-Zustandserkennung beim Einlippen-Tiefbohren. VDI-Z. 144(1/2) 48-51.

Wickerhauser, M. V. (1993). Adapted Wavelet Analysis from Theory to Software. A. K. Peters, Wellesley.

Anita S. Busch

Hella Corporate Center

Corporate Quality Management - Optimization Methods

59552 Lippstadt

Germany

E-mail address: anita.busch@tu-dortmund.de

Ursula Gather

Fakultaet Statistik

Technische Universitaet Dortmund

44221 Dortmund

Germany

E-mail address: gather@statistik.tu-dortmund.de 